\title{
Multiple Solutions to the Problem of Kirchhoff Type Involving the Critical Caffareli-Kohn-Niremberg Exponent, Concave Term and Sign-Changing Weights
}

\author{
Mohammed El Mokhtar Ould El Mokhtar \\ Departement of Mathematics, College of Science, Qassim University, Buraidah, KSA \\ Email: med.mokhtar66@yahoo.fr, M.labdi@qu.edu.sa
}

How to cite this paper: El Mokhtar, M.E.O. (2017) Multiple Solutions to the Problem of Kirchhoff Type Involving the Critical Caffareli-Kohn-Niremberg Exponent, Concave Term and Sign-Changing Weights. Applied Mathematics, 8, 1703-1714.

https://doi.org/10.4236/am.2017.811123

Received: September 26, 2017

Accepted: November 27, 2017

Published: November 30, 2017

Copyright $\odot 2017$ by author and Scientific Research Publishing Inc.

This work is licensed under the Creative

Commons Attribution International

License (CC BY 4.0).

http://creativecommons.org/licenses/by/4.0/ In this paper, we consider the multiplicity results of positive solutions of the (c) (i) Open Access

\begin{abstract}
In this paper, we establish the existence of at least four distinct solutions to an Kirchhoff type problems involving the critical Caffareli-Kohn-Niremberg exponent, concave term and sign-changing weights, by using the Nehari manifold and mountain pass theorem.
\end{abstract}

\section{Keywords}

Kirchhoff Type Problems, Critical Caffareli-Kohn-Niremberg Exponent, Concave Term, Sign-Changing Weights

\section{Introduction} following Kirchhoff problem

$$
\left\{\begin{array}{l}
L_{a, m, n} u=|x|^{-2 * b} f(x)|u|^{2_{*}-2} u+\mu g(x)|u|^{q-2} u \text { in } \mathbb{R}^{3}, x \neq 0 \\
u \in H_{0}^{1}\left(\mathbb{R}^{3}\right),
\end{array}\right.
$$

where $L_{a, m, n}(u)=-\left(m+n \int_{\mathbb{R}^{3}}|x|^{-2 a}|\nabla u|^{2} \mathrm{~d} x\right) \operatorname{div}\left(|x|^{-2 a} \nabla u\right), \quad m>0, n>0, \mu \neq 0$ is a real parameter, $1<q<2,-\infty<a<\frac{1}{2}, a \leq b<a+(1 / 4), \quad 2_{*}=\frac{6}{1+2(b-a)}$ is the critical Caffareli-Kohn-Niremberg exponent and $f, g$ are continuous and sign-changing functions which we will specify later.

The original one-dimensional Kirchhoff equation was introduced by Kirchhoff [1] in 1883 as an generalization of the well-known d'Alembert's wave 
equation:

$$
\rho \frac{\partial^{2} u}{\partial t^{2}}-\left(\frac{P_{0}}{h}+\frac{E}{2 L} \int_{0}^{L}\left|\frac{\partial u}{\partial x}\right|^{2} \mathrm{~d} x\right) \frac{\partial^{2} u}{\partial x^{2}}=g(x ; u) .
$$

His model takes into account the changes in length of the strings produced by transverse vibrations. Here, $L$ is the length of the string, $h$ is the area of the cross section, $E$ is the Young modulus of the material, $\rho$ is the mass density and $P_{0}$ is the initial tension.

In recent years, the existence and multiplicity of solutions to the nonlocal problem

$$
\left\{\begin{array}{l}
-\left(m+n \int_{\Omega}|\nabla u|^{2} d x\right) \Delta u=g(x ; u) \text { in } \Omega, \\
u=0 \text { on } \partial \Omega
\end{array}\right.
$$

has been studied by various researchers and many interesting and important results can be found. In [2], it was pointed out that the problem (1.2) models several physical systems, where $u$ describes a process which depend on the average of itself. Nonlocal effect also finds its applications in biological systems. The movement, modeled by the integral term, is assumed to be dependent on the energy of the entire system with $u$ being its population density. Alternatively, the movement of a particular species may be subject to the total population density within the domain (for instance, the spreading of bacteria) which gives rise to equations of the type

$$
u_{t}-m\left(\int_{\Omega} u \mathrm{~d} x\right) \Delta u=h
$$

For instance, positive solutions could be obtained in [2] [3] [4] [5]. Especially, Chen et al. [6] discussed a Kirchhoff type problem when

$g(x ; u)=f(x) u^{p-2} u+\lambda g(x)|u|^{q-2} u$, where $1<q<2<p<2^{*}=2 N /(N-2)$ if $N \geq 3,2^{*}=\infty$ if $N=1,2, f(x)$ and $g(x)$ with some proper conditions are sign-changing weight functions. And they have obtained the existence of two positive solutions if $p>4,0<\lambda<\lambda_{0}(m)$.

Researchers, such as Mao and Zhang [7], Mao and Luan [8], found sign-changing solutions. As for in nitely many solutions, we refer readers to [9] [10]. He and Zou [11] considered the class of Kirchhoff type problem when $g(x ; u)=\lambda f(x ; u)$ with some conditions and proved a sequence of positive weak solutions tending to zero in $L^{\infty}(\Omega)$.

In the case of a bounded domain of $\mathbb{R}^{N}$ with $N \geq 3$, Tarantello [12] proved, under a suitable condition on $f$, the existence of at least two solutions to (1.2) for $m=0, n=1$ and $g(x ; u)=|u|^{\frac{4}{N-2}} u+f$.

Before formulating our results, we give some definitions and notation.

The space $\mathcal{H}=\mathcal{H}_{0}^{1}\left(\mathbb{R}^{3}\right)$ is equiped with the norm

$$
\|u\|=\left(\int_{\mathbb{R}^{3}}|x|^{-2 a}|\nabla u|^{2} \mathrm{~d} x\right)^{1 / 2} .
$$

Let $S_{\mu}$ be the best Sobolev constant, then 


$$
S_{a}=\inf _{u \in \mathcal{H}_{0}^{1}\left(\mathbb{R}^{3}\right)\{0\}} \frac{\int_{\mathbb{R}^{3}}|x|^{-2 a}|\nabla u|^{2} \mathrm{~d} x}{\left(\int_{\mathbb{R}^{3}}|x|^{-2 * b} f|u|^{2_{*}^{2}} \mathrm{~d} x\right)^{\frac{2}{2_{*}}}}
$$

Since our approach is variational, we define the functional $J$ on $\mathcal{H}_{0}^{1}\left(\mathbb{R}^{3}\right)$ by

$$
\begin{aligned}
J(u)= & (1 / 2) m\|u\|^{2}+(1 / 4) n\|u\|^{4} \\
& -\left(1 / 2_{*}\right) \int_{\mathbb{R}^{3}}|x|^{-2_{*} b} f|u|^{2_{*}} \mathrm{~d} x-(\mu / q) \int_{\mathbb{R}^{3}} g|u|^{q} \mathrm{~d} x,
\end{aligned}
$$

A point $u \in \mathcal{H}_{0}^{1}\left(\mathbb{R}^{3}\right)$ is a weak solution of the Equation (1.1) if it is the critical point of the functional $J$. Generally speaking, a function $u$ is called a solution of (1.1) if $u \in \mathcal{H}_{0}^{1}\left(\mathbb{R}^{3}\right)$ and for all $v \in \mathcal{H}_{0}^{1}\left(\mathbb{R}^{3}\right)$ it holds

$$
\begin{aligned}
& \left(m+n\|u\|^{2}\right) \int_{\mathbb{R}^{3}}\left(|x|^{-2 a} \nabla u \nabla v\right) \mathrm{d} x-\int_{\mathbb{R}^{3}}|x|^{-2_{*} b} f|u|^{2_{*}-2} u v \mathrm{~d} x \\
& -\mu \int_{\mathbb{R}^{3}} g|u|^{q-2} u v \mathrm{~d} x=0 .
\end{aligned}
$$

Throughout this work, we consider the following assumptions:

(F) $f$ is a continuous function satisfies:

$$
f(0)=\max _{x \in \mathbb{R}^{3}} f(x)>0, f(x)=f(0)+o\left(x^{\beta}\right), \text { with } \beta>\frac{3(1-2 a)}{1+2(b-a)},
$$

(G) $h$ is a continuous function and there exist $g_{0}$ and $\rho_{0}$ positive such that:

$$
g(x) \geq g_{0} \text { for all } x \in B\left(0,2 \rho_{0}\right) .
$$

Here, $B(a, r)$ denotes the ball centered at $a$ with radius $r$.

In our work, we research the critical points as the minimizers of the energy functional associated to the problem (1.1) on the constraint defined by the Nehari manifold, which are solutions of our problem.

Let $\mu_{0}$ be real number such that

$$
\mu_{0}=\frac{2 n(A+B)}{\left(2_{*}-q\right)\left|g^{+}\right|_{\infty}} X_{0}^{\frac{2-q}{2_{*}-q}}-\frac{2 n A^{\prime}}{\left(2_{*}-q\right)\left|g^{+}\right|_{\infty}} X_{0}^{\frac{2_{*}-q}{2_{*}-q}},
$$

where

$$
A=\frac{2 m}{n}, B=\frac{(q-2) m}{(4-q) n}, A^{\prime}=\left(\frac{2_{*}-q}{4-q}\right) \frac{\left(S_{a}\right)^{\frac{2}{2 *}}}{n}, X_{0}=\left[\frac{\left(2_{*}-q\right) A}{(2-q)\left(A^{\prime}+B\right)}\right]^{\frac{1}{2-2_{*}}} .
$$

Now we can state our main results.

Theorem 1 Assume that $1<q<2,-\infty<a<\frac{1}{2}, a \leq b<a+(1 / 4)$, and $(F)$ satisfied and $\mu$ verifying $\mu<\mu_{0}$, then the problem (1.1) has at least one positive solution.

Theorem 2 In addition to the assumptions of the Theorem 1, if $(G)$ hold, then there exists $\mu_{1}>0$ such that for all $\mu$ verifying $0<\mu<\min \left(\mu_{0}, \mu_{1}\right)$ the problem (1.1) has at least two positive solutions.

Theorem 3 In addition to the assumptions of the Theorem 2, assuming 
$\mu<0$, then the problem (1.1) has at least two positive solution and two opposite solutions.

This paper is organized as follows. In Section 2, we give some preliminaries. Section 3 and 4 are devoted to the proofs of Theorems 1 and 2. In the last Section, we prove the Theorem 3.

\section{Preliminaries}

Definition 1 Let $c \in \mathbb{R}, E$ a Banach space and $I \in C^{1}(E, \mathbb{R})$.

1) $\left(u_{n}\right)_{n}$ is a Palais-Smale sequence at level $c$ (in short $(P S)_{c}$ ) in $E$ for $I$ if

$$
I\left(u_{n}\right)=c+o_{n}(1) \text { and } I^{\prime}\left(u_{n}\right)=o_{n}(1),
$$

where $o_{n}(1)$ tends to 0 as $n$ goes at infinity.

2) We say that $I$ satisfies the $(P S)_{c}$ condition if any $(P S)_{c}$ sequence in $E$ for $I$ has a convergent subsequence.

Lemma 1 Let $X$ Banach space, and $J \in C^{1}(X, \mathbb{R})$ verifying the Palais-Smale condition. Suppose that $J(0)=0$ and that:

1) there exist $R>0, r>0$ such that if $\|u\|=R$, then $J(u) \geq r$;

2) there exist $\left(u_{0}\right) \in X$ such that $\left\|u_{0}\right\|>R$ and $J\left(u_{0}\right) \leq 0$; let $c=\inf _{\gamma \in \Gamma \in[0,1]}(J(\gamma(t)))$ where

$$
\Gamma=\left\{\gamma \in C([0,1] ; X) \text { such that } \gamma(0)=0 \text { et } \gamma(1)=u_{0}\right\},
$$

then $c$ is critical value of $J$ such that $c \geq r$.

\section{Nehari Manifold}

It is well known that the functional $J$ is of class $C^{1}$ in $\mathcal{H}_{0}^{1}\left(\mathbb{R}^{3}\right)$ and the solutions of (1.1) are the critical points of $J$ which is not bounded below on $\mathcal{H}_{0}^{1}\left(\mathbb{R}^{3}\right)$. Consider the following Nehari manifold

$$
\mathcal{N}=\left\{u \in \mathcal{H}_{0}^{1}\left(\mathbb{R}^{3}\right) \backslash\{0\}:\left\langle J^{\prime}(u), u\right\rangle=0\right\},
$$

Thus, $u \in \mathcal{N}$ if and only if

$$
\left(m+n\|u\|^{2}\right) \int_{\mathbb{R}^{3}}\left(|x|^{-2 a}|\nabla u|^{2}\right) \mathrm{d} x-\int_{\mathbb{R}^{3}}|x|^{-2 * b} f|u|^{2^{*}} \mathrm{~d} x-\mu \int_{\mathbb{R}^{3}} g|u|^{q} \mathrm{~d} x=0
$$

Define

$$
\varphi(u)=\left\langle J^{\prime}(u), u\right\rangle .
$$

Then, for $u \in \mathcal{N}$

$$
\begin{aligned}
\left\langle\varphi^{\prime}(u), u\right\rangle & =\left(2 m+4 n\|u\|^{2}\right)\|u\|^{2}-2 \int_{\mathbb{R}^{3}}|x|^{-2_{*} b} f|u|^{2 *} \mathrm{~d} x-\mu q \int_{\Omega^{2}} g|u|^{q} \mathrm{~d} x \\
& =\left[(2-q) m+(4-q) n\|u\|^{2}\right]\|u\|^{2}-\left(2_{*}-q\right) \int_{\mathbb{R}^{3}}|x|^{-2_{*} b} f|u|^{2_{*}} \mathrm{~d} x \\
& =\left(2_{*}-q\right) \mu \int_{\mathbb{R}^{3}} g|u|^{q} \mathrm{~d} x-\left(4 m+2 n\|u\|^{2}\right)\|u\|^{2} .
\end{aligned}
$$

Now, we split $\mathcal{N}$ in three parts:

$$
\mathcal{N}^{+}=\left\{u \in \mathcal{N}:\left\langle\varphi^{\prime}(u), u\right\rangle>0\right\}
$$




$$
\begin{aligned}
\mathcal{N}^{0} & =\left\{u \in \mathcal{N}:\left\langle\varphi^{\prime}(u), u\right\rangle=0\right\} \\
\mathcal{N}^{-} & =\left\{u \in \mathcal{N}:\left\langle\varphi^{\prime}(u), u\right\rangle<0\right\} .
\end{aligned}
$$

Note that $\mathcal{N}$ contains every nontrivial solution of the problem (1.1). Moreover, we have the following results.

Lemma $2 J$ is coercive and bounded from below on $\mathcal{N}$.

Proof. If $u \in \mathcal{N}$, then by (2.3) and the Hölder inequality, we deduce that

$$
\begin{aligned}
J(u) & =(1 / 2) m\|u\|^{2}+(1 / 4) n\|u\|^{4}-\left(1 / 2_{*}\right) \int_{\mathbb{R}^{3}}|x|^{-2_{*} b} f|u|^{2 *} \mathrm{~d} x-(\mu / q) \int_{\mathbb{R}^{3}} g|u|^{q} \mathrm{~d} x, \\
& \geq m\left(\frac{1}{2}-\frac{1}{2_{*}}\right)\|u\|^{2}+n\left(\frac{1}{4}-\frac{1}{2_{*}}\right)\|u\|^{4}-\mu\left(\frac{1}{q}-\frac{1}{2_{*}}\right)\left|g^{+}\right|_{\infty}\|u\|^{q} .
\end{aligned}
$$

Thus, $J$ is coercive and bounded from below on $\mathcal{N}$.

We have the following results.

Lemma 3 Suppose that $u_{0}$ is a local minimizer for $J$ on $\mathcal{N}$. Then, if $u_{0} \notin \mathcal{N}^{0}, u_{0}$ is a critical point of $)$.

Proof. If $u_{0}$ is a local minimizer for $J$ on $\mathcal{N}$, then $u_{0}$ is a solution of the optimization problem

$$
\min _{\{u / \varphi(u)=0\}} J(u) .
$$

Hence, there exists a Lagrange multipliers $\theta \in \mathbb{R}$ such that

$$
J^{\prime}\left(u_{0}\right)=\theta \varphi^{\prime}\left(u_{0}\right) \text { in } \mathcal{H}^{-1}
$$

Thus,

$$
\left\langle J^{\prime}\left(u_{0}\right), u_{0}\right\rangle=\theta\left\langle\varphi^{\prime}\left(u_{0}\right), u_{0}\right\rangle .
$$

But $\left\langle\varphi^{\prime}\left(u_{0}\right), u_{0}\right\rangle \neq 0$, since $u_{0} \notin \mathcal{N}^{0}$. Hence $\theta=0$. This completes the proof.

Lemma 4 There exists a positive number $\mu_{0}$ such that, for all $\mu \in\left(0, \mu_{0}\right)$ we have $\mathcal{N}^{0}=\varnothing$.

Proof. Let us reason by contradiction.

Suppose $\mathcal{N}^{0} \neq \varnothing$ such that $0<\mu<\mu_{0}$. Moreover, by the Hölder inequality and the Sobolev embedding theorem, we obtain

$$
\|u\|^{4} \geq A^{\prime}\|u\|^{2}-B^{\prime}\|u\|^{q}
$$

and

$$
\|u\|_{\mu}^{4} \leq A\|u\|^{2 *}-B\|u\|^{2}
$$

with

$$
A=\left(\frac{2_{*}-q}{4-q}\right) \frac{\left(S_{a}\right)^{\frac{2}{2}}}{n}, B=\left(\frac{2-q}{6-q}\right)\left(\frac{m}{n}\right), A^{\prime}=\frac{2 m}{n}, B^{\prime}=\left(\frac{2_{*}-q}{2 n}\right) \mu\left|g^{+}\right|_{\infty} .
$$

From (2.5) and (2.6), we obtain $\mu \geq \mu_{0}$, which contradicts an hypothesis.

Thus $\mathcal{N}=\mathcal{N}^{+} \cup \mathcal{N}^{-}$. Define

$$
\delta:=\inf _{u \in \mathcal{N}} J(u), \delta^{+}:=\inf _{u \in \mathcal{N}^{+}} J(u) \text { and } \delta^{-}:=\inf _{u \in \mathcal{N}^{-}} J(u) \text {. }
$$


For the sequel, we need the following Lemma.

\section{Lemma 5}

1) For all $\mu$ such that $0<\mu<\mu_{0}$, one has $\delta \leq \delta^{+}<0$.

2) There exists $\mu_{1}>0$ such that for all $0<\mu<\mu_{1}$, one has

$$
\delta^{-}>C_{0}=C_{0}\left(m, n, q,\left|g^{+}\right|_{\infty}\right) \text {. }
$$

Proof. 1) Let $u \in \mathcal{N}^{+}$. By (2.4), we have

$$
\left[\left((2-q) m+(4-q) n\|u\|^{2}\right) /\left(2_{*}-q\right)\right]\|u\|^{2}>\int_{\mathbb{R}^{3}}|x|^{-2_{*} b} f|u|^{2_{*}} \mathrm{~d} x
$$

and so

$$
\begin{aligned}
J(u)= & \left(\frac{1}{4}-\frac{1}{q}\right) n\|u\|^{4}+\left(\frac{1}{2}-\frac{1}{q}\right) m\|u\|^{2}+\left(\frac{2 *}{q}-\frac{1}{2_{*}}\right) \int_{\mathbb{R}^{3}}|x|^{-2 * b} f|u|^{2 *} \mathrm{~d} x \\
< & -\left[\left(\frac{1}{4}-\frac{1}{q}\right)-\left(\frac{2 *}{q}-\frac{1}{2_{*}}\right)\left(\frac{4-q}{2_{*}-q}\right)\right] n\|u\|^{4} \\
& -\left[\left(\frac{1}{q}-\frac{1}{2}\right)-\left(\frac{2 *}{q}-\frac{1}{2_{*}}\right)\left(\frac{2-q}{2_{*}-q}\right)\right] m\|u\|^{2}
\end{aligned}
$$

We conclude that $\delta \leq \delta^{+}<0$.

2) Let $u \in \mathcal{N}^{-}$. By (2.4) and the Hölder inequality we get

$$
\begin{aligned}
J(u) & =m\left(\frac{1}{2}-\frac{1}{2_{*}}\right)\|u\|^{2}+n\left(\frac{1}{4}-\frac{1}{2_{*}}\right)\|u\|_{\mu}^{4}-\mu \int_{\mathbb{R}^{3}} g|u|^{q} \mathrm{~d} x \\
& \geq m\left(\frac{1}{2}-\frac{1}{2_{*}}\right)\|u\|^{2}-\mu\left(\frac{1}{q}-\frac{1}{2_{*}}\right)\left\|\left.g^{+}\right|_{\infty}\right\| u \|^{q} .
\end{aligned}
$$

Thus, for all $\mu$ such that $0<\mu<\mu_{1}=\frac{m\left(\frac{1}{2}-\frac{1}{2_{*}}\right)}{\left(\frac{1}{q}-\frac{1}{2_{*}}\right)\left|g^{+}\right|_{\infty}}$, we have $J(u) \geq C_{0}$.

We define:

$$
\begin{aligned}
& F^{+}:=\left\{u \in \mathcal{N} / \int_{\mathbb{R}^{3}}|x|^{-2_{*} b} f|u|^{2_{*}} \mathrm{~d} x>0\right\}, G^{+}:=\left\{u \in \mathcal{N} / \int_{\mathbb{R}^{3}} g|u|^{q} d x>0\right\}, \\
& F_{0}^{-}:=\left\{u \in \mathcal{N} / \int_{\mathbb{R}^{3}}|x|^{-2_{*} b} f|u|^{2_{*}} \mathrm{~d} x \leq 0\right\}, G_{0}^{-}:=\left\{u \in \mathcal{N} / \int_{\mathbb{R}^{3}} g|u|^{q} d x \leq 0\right\},
\end{aligned}
$$

and for each $u \in \mathcal{H}$ with $u \in F^{+}$, we write

$$
t_{m}:=t_{\max }(u)=\left[\frac{8 n\left(\frac{1}{q}-\frac{1}{4}\right)}{22_{*}\left(2_{*}-2\right)\left(\frac{2_{*}}{q}-\frac{1}{2_{*}}\right) \int_{\mathbb{R}^{3}}|x|^{-2_{*} b} f|u|^{2_{*}} \mathrm{~d} x}\right]^{\frac{1}{2_{*}-4}}>0 .
$$

Lemma 6 Let $\mu$ real parameters such that $0<\mu<\mu_{0}$. For each $u \in \mathcal{H}$ we have:

1) If $u \in F^{+} \cap G_{0}^{-}$then there exists unique $t^{+}>t_{M}$ such that $t^{+} u \in \mathcal{N}^{-}$and

$$
J\left(t^{+} u\right) \geq J(t u) \text { for } t>t_{M},
$$


2) If $u \in F^{+} \cap G^{+}$then there exist unique $t^{+}$and $t^{-}$such that $0<t^{-}<t_{M}<t^{+},\left(t^{+} u\right) \in \mathcal{N}^{-}, t^{-} u \in \mathcal{N}^{+}$and

$$
J\left(t^{+} u\right) \geq J(t u) \text { for } t \geq t^{-} \text {and } J\left(t^{-} u\right) \leq J(t u) \text { for } t \in\left[0, t^{+}\right]
$$

3) If $u \in F^{-} \cap G^{-}$, then does not exist $t>0$ such that $(t u) \in \mathcal{N}$.

4) If $u \in F_{0}^{-} \cap G^{+}$, then there exists unique $0<t^{-}<+\infty$ such that $\left(t^{-} u\right) \in \mathcal{N}^{+}$

and

$$
J\left(t^{-} u\right)=\inf _{t \geq 0} J(t u) .
$$

Proof. With minor modifications, we refer to [13].

Proposition 1 (see [13)

1) For all $\mu$ such that $0<\mu<\mu_{0}$, there exists a $(P S)_{\delta^{+}}$sequence in $\mathcal{N}^{+}$.

2) For all $\mu$ such that $0<\mu<\mu_{1}$, there exists a a $(P S)_{\delta^{-}}$sequence in $\mathcal{N}^{-}$.

\section{Proof of Theorems 1}

Now, taking as a starting point the work of Tarantello [12], we establish the existence of a local minimum for Jon $\mathcal{N}^{+}$.

Proposition 2 For all $\mu$ such that $0<\mu<\mu_{0}$, the functional $J$ has a minimizer $u_{0}^{+} \in \mathcal{N}^{+}$and it satisfies:

1) $J\left(u_{0}^{+}\right)=\delta=\delta^{+}$

2) $\left(u_{0}^{+}\right)$is a nontrivial solution of (1.1).

Proof. If $0<\mu<\mu_{0}$, then by Proposition 1 (i) there exists a $\left(u_{n}\right)_{n}(P S)_{\delta^{+}}$ sequence in $\mathcal{N}^{+}$, thus it bounded by Lemma 2 . Then, there exists $u_{0}^{+} \in \mathcal{H}$ and we can extract a subsequence which will denoted by $\left(u_{n}\right)_{n}$ such that

$$
\begin{gathered}
u_{n} \rightarrow u_{0}^{+} \text {weakly in } \mathcal{H}_{0}^{1}\left(\mathbb{R}^{3}\right) \\
u_{n} \rightarrow u_{0}^{+} \text {weakly in } L^{2 *}\left(\mathbb{R}^{3}\right) \\
u_{n} \rightarrow u_{0}^{+} \text {strongly in } L^{q}\left(\mathbb{R}^{3}\right) \\
u_{n} \rightarrow u_{0}^{+} \text {a.e in } \mathbb{R}^{3}
\end{gathered}
$$

Thus, by (3.1), $u_{0}^{+}$is a weak nontrivial solution of (1.1). Now, we show that $u_{n}$ converges to $u_{0}^{+}$strongly in $\mathcal{H}_{0}^{1}\left(\mathbb{R}^{3}\right)$. Suppose otherwise. By the lower semi-continuity of the norm, then either $\left\|u_{0}^{+}\right\|<\liminf _{n \rightarrow \infty}\left\|u_{n}\right\|$ and we obtain

$$
\begin{aligned}
\delta & \leq J\left(u_{0}^{+}\right)=m\left(\frac{1}{2}-\frac{1}{2_{*}}\right)\left\|u_{0}^{+}\right\|^{2}+n\left(\frac{1}{4}-\frac{1}{2_{*}}\right)\left\|u_{0}^{+}\right\|^{4}-\mu \int_{\mathbb{R}^{3}} g\left|u_{0}^{+}\right|^{q} \mathrm{~d} x \\
& <\liminf _{n \rightarrow \infty} J\left(u_{n}\right)=\delta .
\end{aligned}
$$

We get a contradiction. Therefore, $u_{n}$ converge to $u_{0}^{+}$strongly in $\mathcal{H}_{0}^{1}\left(\mathbb{R}^{3}\right)$. Moreover, we have $u_{0}^{+} \in \mathcal{N}^{+}$. If not, then by Lemma 6 , there are two numbers $t_{0}^{+}$and $t_{0}^{-}$, uniquely defined so that $\left(t_{0}^{+} u_{0}^{+}\right) \in \mathcal{N}^{+}$and $\left(t^{-} u_{0}^{+}\right) \in \mathcal{N}^{-}$. In 
particular, we have $t_{0}^{+}<t_{0}^{-}=1$. Since

$$
\left.\frac{\mathrm{d}}{\mathrm{d} t} J\left(t u_{0}^{+}\right)\right|_{t=t_{0}^{+}}=0 \text { and }\left.\frac{\mathrm{d}^{2}}{\mathrm{~d} t^{2}} J\left(t u_{0}^{+}\right)\right|_{t=t_{0}^{+}}>0 \text {, }
$$

there exists $t_{0}^{+}<t^{-} \leq t_{0}^{-}$such that $J_{\lambda}\left(t_{0}^{+} u_{0}^{+}\right)<J_{\lambda}\left(t^{-} u_{0}^{+}\right)$. By Lemma 6, we get

$$
J_{\lambda}\left(t_{0}^{+} u_{0}^{+}\right)<J_{\lambda}\left(t^{-} u_{0}^{+}\right)<J_{\lambda}\left(t_{0}^{-} u_{0}^{+}\right)=J_{\lambda}\left(u_{0}^{+}\right),
$$

which contradicts the fact that $J\left(u_{0}^{+}\right)=c^{+}$. Since $J\left(u_{0}^{+}\right)=J\left(\left|u_{0}^{+}\right|\right)$and $\left|u_{0}^{+}\right| \in \mathcal{N}^{+}$, then by Lemma 6, we may assume that $u_{0}^{+}$is a nontrivial nonnegative solution of (1.1). By the Harnack inequality, we conclude that $u_{0}^{+}>0$, see for exanmple [14].

\section{Proof of Theorem 2}

Next, we establish the existence of a local minimum for $J$ on $\mathcal{N}^{-}$. For this, we require the following Lemma.

Lemma 7 Assume that $n>\frac{3(6-q)}{(4-q)}$ then, for all $\mu$ such that $0<\mu<\mu_{1}$, the functional $J$ has a minimizer $u_{0}^{-}$in $\mathcal{N}^{-}$and it satisfies:

1) $J\left(u_{0}^{-}\right)=\delta^{-}>0$,

2) $u_{0}^{-}$is a nontrivial solution of $(1.1)$ in $\mathcal{H}_{0}^{1}\left(\mathbb{R}^{3}\right)$.

Proof. If $0<\delta<\delta_{1}$, then by Proposition 1 (ii) there exists a $\left(u_{n}\right)_{n},(P S)_{\delta^{-}}$ sequence in $\mathcal{N}^{-}$, thus it bounded by Lemma 2 . Then, there exists $u_{0}^{-} \in \mathcal{H}_{0}^{1}\left(\mathbb{R}^{3}\right)$ and we can extract a subsequence which will denoted by $\left(u_{n}\right)_{n}$ such that

$$
\begin{gathered}
u_{n} \rightarrow u_{0}^{-} \text {weakly in } \mathcal{H}_{0}^{1}\left(\mathbb{R}^{3}\right) \\
u_{n} \rightarrow u_{0}^{-} \text {weakly in } L^{2 *}\left(\mathbb{R}^{3}\right) \\
u_{n} \rightarrow u_{0}^{-} \text {strongly in } L^{q}\left(\mathbb{R}^{3}\right) \\
u_{n} \rightarrow u_{0}^{-} \text {a.e in } \mathbb{R}^{3}
\end{gathered}
$$

This implies that

$$
\int_{\mathbb{R}^{3}}|x|^{-2_{*} b} f\left|u_{n}\right|^{2_{*}} \mathrm{~d} x \rightarrow \int_{\mathbb{R}^{3}}|x|^{-2_{*} b} f\left|u_{0}^{-}\right|^{2_{*}} \mathrm{~d} x \text {, as } n \text { goes to } \infty .
$$

Moreover, by (G) and (2.4) we obtain

$$
\begin{aligned}
\int_{\mathbb{R}^{3}}|x|^{-2_{*} b} f\left|u_{n}\right|^{2_{*}} \mathrm{~d} x & >\left[\frac{(4-q)}{\left(2_{*}-q\right)} n\left\|u_{n}\right\|^{4}+\frac{(2-q)}{\left(2_{*}-q\right)} m\left\|u_{n}\right\|^{2}\right] \\
& >\left[\frac{(4-q)}{\left(2_{*}-q\right)} n\left\|u_{n}\right\|^{4}+\frac{(2-q)}{\left(2_{*}-q\right)} m\left\|u_{n}\right\|^{2}\right]-\left\|u_{n}\right\|^{2} \\
& >C_{1}=\left[\frac{(2-q)}{\left(2_{*}-q\right)} m\right]^{2}\left[\frac{(4-q)}{\left(2_{*}-q\right)} n-3\right]\left[\frac{2(4-q)}{\left(2_{*}-q\right)} n-2\right]^{-2}
\end{aligned}
$$

if $n>\frac{3(6-q)}{(4-q)}$ we get

$$
\int_{\mathbb{R}^{3}}|x|^{-2 * b} f\left|u_{n}\right|^{2 *} \mathrm{~d} x>C_{1}>0
$$


This implies that

$$
\int_{\mathbb{R}^{3}}|x|^{-2_{*} b} f\left|u_{0}^{-}\right|^{2 *} \mathrm{~d} x \geq C_{1} .
$$

Now, we prove that $\left(u_{n}\right)_{n}$ converges to $u_{0}^{-}$strongly in $\mathcal{H}_{0}^{1}\left(\mathbb{R}^{3}\right)$. Suppose otherwise. Then, either $\left\|u_{0}^{-}\right\|<\liminf _{n \rightarrow \infty}\left\|u_{n}\right\|$. By Lemma 6 there is a unique $t_{0}^{-}$ such that $\left(t_{0}^{-} u_{0}^{-}\right) \in \mathcal{N}^{-}$. Since

$$
u_{n} \in \mathcal{N}^{-}, J\left(u_{n}\right) \geq J\left(t u_{n}\right), \text { for all } t \geq 0,
$$

we have

$$
J\left(t_{0}^{-} u_{0}^{-}\right)<\lim _{n \rightarrow \infty} J\left(t_{0}^{-} u_{n}\right) \leq \lim _{n \rightarrow \infty} J\left(u_{n}\right)=\delta^{-},
$$

and this is a contradiction. Hence,

$$
\left(u_{n}\right)_{n} \rightarrow u_{0}^{-} \text {strongly in } \mathcal{H}_{0}^{1}\left(\mathbb{R}^{3}\right) \text {. }
$$

Thus,

$$
J\left(u_{n}\right) \text { converges to } J\left(u_{0}^{-}\right)=\delta^{-} \text {as } n \text { tends to }+\infty .
$$

Since $J\left(u_{0}^{-}\right)=J\left(\left|u_{0}^{-}\right|\right)$and $u_{0}^{-} \in \mathcal{N}^{-}$, then by (4.1) and Lemma 3, we may assume that $u_{0}^{-}$is a nontrivial nonnegative solution of (1.1). By the maximum principle, we conclude that $u_{0}^{-}>0$.

Now, we complete the proof of Theorem 2. By Propositions 2 and Lemma 7, we obtain that (1.1) has two positive solutions $u_{0}^{+} \in \mathcal{N}^{+}$and $u_{0}^{-} \in \mathcal{N}^{-}$. Since $\mathcal{N}^{+} \cap \mathcal{N}^{-}=\varnothing$, this implies that $u_{0}^{+}$and $u_{0}^{-}$are distinct.

\section{Proof of Theorem 3}

In this section, we consider the following Nehari submanifold of $\mathcal{N}$

$$
\mathcal{N}_{\rho}=\left\{u \in \mathcal{H}_{0}^{1}\left(\mathbb{R}^{3}\right) \backslash\{0\}:\left\langle J^{\prime}(u), u\right\rangle=0 \text { and }\|u\| \geq \rho>0\right\} .
$$

Thus, $u \in \mathcal{N}_{\rho}$ if and only if

$$
\begin{aligned}
& \left(m+n\|u\|^{2}\right) \int_{\mathbb{R}^{3}}\left(|x|^{-2 a}|\nabla u|^{2}\right) \mathrm{d} x-\int_{\mathbb{R}^{3}}|x|^{-2_{*} b} f|u|^{2 *} \mathrm{~d} x \\
& -\mu \int_{\mathbb{R}^{3}} g|u|^{q} \mathrm{~d} x=0 \text { and }\|u\| \geq \rho>0 .
\end{aligned}
$$

Firsly, we need the following Lemmas

Lemma 8 Under the hypothesis of theorem 3, there exist $\rho_{0}$ such that $\mathcal{N}_{\rho}$ is nonempty for any $\mu<0$ and $\rho \in\left(0, \rho_{0}\right)$.

Proof. Fix $u_{0} \in \mathcal{H}_{0}^{1}\left(\mathbb{R}^{3}\right) \backslash\{0\}$ and let

$$
\begin{aligned}
g(t) & =\left\langle J^{\prime}\left(t u_{0}\right), t u_{0}\right\rangle \\
& =m t^{2}\left\|\left.u_{0}\right|^{2}+n t^{4}\right\| u_{0} \|^{4}-t^{2 *} \int_{\mathbb{R}^{3}}|x|^{-2_{*} b} f\left|u_{0}\right|^{2 *} \mathrm{~d} x-\mu t^{q} \int_{\mathbb{R}^{3}} g\left|u_{0}\right|^{q} \mathrm{~d} x .
\end{aligned}
$$

Clearly $g(0)=0$ and $g(t) \rightarrow-\infty$ as $n \rightarrow+\infty$. Moreover, we have

$$
g(1)=m\left\|u_{0}\right\|^{2}+n\left\|u_{0}\right\|^{4}-\int_{\mathbb{R}^{3}}|x|^{-2_{*} b} f\left|u_{0}\right|^{2_{*}} \mathrm{~d} x-\mu \int_{\mathbb{R}^{3}} g\left|u_{0}\right|^{q} \mathrm{~d} x .
$$

If $\left\|u_{0}\right\|_{\mu} \geq \rho>0$ for $0<\rho<\rho_{0}=\left(m\left|g^{+}\right|_{\infty}^{-1}\right)^{1 / 4}\left(S_{a}\right)^{3 / 4}$, then there exists $t_{0}>0$ 
such that $g\left(t_{0}\right)=0$. Thus, $\left(t_{0} u_{0}\right) \in \mathcal{N}_{\rho}$ and $\mathcal{N}_{\rho}$ is nonempty for any $\mu<0$.

Lemma 9 There exist $M$ positive real such that

$$
\left\langle\varphi^{\prime}(u), u\right\rangle<-M<0,
$$

for $u \in \mathcal{N}_{\rho}$ and any $\mu<0$.

Proof. Let $u \in \mathcal{N}_{\rho}$, then by (2.3), (2.4) and the Holder inequality, allows us to write

$$
\left\langle\varphi^{\prime}(u), u\right\rangle \leq \mu\left(2_{*}-q\right)\|u\|^{2}\left|g^{+}\right|_{\infty}-\left(4 m+2 n\|u\|^{2}\right)\|u\|^{2} .
$$

Thus, if $\mu<0$ then we obtain that

$$
\left\langle\varphi^{\prime}(u), u\right\rangle<0 \text {, for any } u \in \mathcal{N}_{\rho} .
$$

Lemma 10 There exist $r$ and $\eta$ positive constants such that

1) we have

$$
J(u) \geq \eta>0 \text { for }\|u\|=r .
$$

2) there exists $\sigma \in \mathcal{N}_{\rho}$ when $\|\sigma\|>r$, with $r=\|u\|$, such that $J(\sigma) \leq 0$.

Proof. We can suppose that the minima of $J$ are realized by $\left(u_{0}^{+}\right)$and $u_{0}^{-}$. The geometric conditions of the mountain pass theorem are satisfied. Indeed, we have

1) By (2.4), (5.1), the Holder inequality, we get

$$
J(u) \geq m\left(\frac{1}{2}-\frac{1}{2_{*}}\right)\|u\|^{2}-\mu\left(\frac{1}{q}-\frac{1}{2_{*}}\right)\left\|\left.g^{+}\right|_{\infty}\right\| u \|^{q} .
$$

Thus, for $\mu<0$ there exist $\eta, r>0$ such that

$$
J(u) \geq \eta>0 \text { when } r=\|u\| \text { small. }
$$

2) Let $t>0$, then we have for all $\theta \in \mathcal{N}_{\rho}$

$$
J(t \theta)=\frac{m}{2} t^{2}\|\theta\|^{2}+\frac{n}{4} t^{4} \|\left.\theta\right|^{4}-\frac{1}{2_{*}} t^{2 *} \int_{\Omega} h|\theta|^{6} \mathrm{~d} x-\frac{\mu}{q} t^{q} \int_{\Omega} f|\theta|^{q} \mathrm{~d} x .
$$

By the fact that $b<a+\frac{1}{4}$ we have $2_{*}>4$ and letting $\sigma=t \theta$ for $t$ large enough, we obtain $J(\sigma) \leq 0$. For $t$ large enough we can ensure $\|\sigma\|>r$.

Let $\Gamma$ and $\delta$ defined by

$$
\Gamma:=\left\{\gamma:[0,1] \rightarrow \mathcal{N}_{\rho}: \gamma(0)=u_{0}^{-} \text {and } \gamma(1)=u_{0}^{+}\right\}
$$

and

$$
\delta:=\inf _{\gamma \in \Pi} \max _{t \in[0,1]}(J(\gamma(t)))
$$

\section{Proof of Theorem 3.}

If $\mu<0$ then, by the Lemmas 2 and Proposition 12 ), $J$ verifying the Palais -Smale condition in $\mathcal{N}_{\rho}$. Moreover, from the Lemmas 3, 9 and 10, there exists $u_{\delta}$ such that

$$
J\left(u_{\delta}\right)=\delta \text { and } u_{\delta} \in \mathcal{N}_{\rho} .
$$


Thus $u_{\delta}$ is the third solution of our system such that $u_{\delta} \neq u_{0}^{+}$and $u_{\delta} \neq u_{0}^{-}$. Since (1.1) is odd with respect $u$, we obtain that $-u_{\delta}$ is also a solution of (1.1).

\section{Acknowledgements}

The author gratefully acknowledges Qassim University, represented by the Deanship of Scientific Research, on the material support for this research under the number (1026) during the academic year 1438AH/2017AD.

\section{References}

[1] Kirchhoff, G. (1883) Vorlesungen über Mechanik. Teubner, Leipzig.

[2] Alves C.O., Correa, F.J.S.A. and Ma, T.F. (2005) Positive Solutions for a Quasilinear Elliptic Equation of Kirchhoff Type. Computers \& Mathematics with Applications, 49, 85-93. https://doi.org/10.1016/j.camwa.2005.01.008

[3] Cheng, C.T. and Wu, X. (2009) Existence Results of Positive Solutions of Kirchhoff Type Problems. Nonlinear Analysis, 71, 4883-4892. https://doi.org/10.1016/j.na.2009.03.065

[4] Li, G.B. and Ye, H.Y. (2014) Existence of Positive Ground State Solutions for the Nonlinear Kirchhoff Type Equations in $\mathrm{R}^{3}$. Journal of Differential Equations, 257, 566-600.

[5] Ma, T.F. and Rivera, J.E.M. (2003) Positive Solutions for a Nonlinear Nonlocal Elliptic Transmission Problem. Applied Mathematics Letters, 16, 243-248. https://doi.org/10.1016/S0893-9659(03)80038-1

[6] Chen C., Kuo, Y. and Wu, T. (2011) The Nehari Manifold for a Kirchhoff Type Problem Involving Sign Changing Weight Functions. Journal of Differential Equations, 250, 1876-1908.

[7] Mao, A.M. and Zhang, Z.T. (2009) Sign-Changing and Multiple Solutions of Kirchhoff Type Problems without the P.S. Condition. Nonlinear Analysis, 70, 1275-1287. https://doi.org/10.1016/j.na.2008.02.011

[8] Mao, A.M. and Luan, S.X. (2011) Sign-Changing Solutions of a Class of Nonlocal Quasilinear Elliptic Boundary Value Problems. Journal of Mathematical Analysis and Applications, 383, 239-243. https://doi.org/10.1016/j.jmaa.2011.05.021

[9] Jin, J.H. and Wu, X. (2010) Innitely Many Radial Solutions for Kirchhoff-Type Problems in $\mathrm{R}^{\mathrm{N}}$. Journal of Mathematical Analysis and Applications, 369, 564-574. https://doi.org/10.1016/j.jmaa.2010.03.059

[10] Wei, L. and He, X M. (2012) Multiplicity of High Energy Solutions for Superlinearkirchho Equations. Journal of Applied Mathematics and Computing, 39, 473-487. https://doi.org/10.1007/s12190-012-0536-1

[11] He, X.M. and Zou, W.M. (2009) Innitely Many Positive Solutions for Kirchhoff-Type Problems. Nonlinear Analysis, 70, 1407-1414. https://doi.org/10.1016/j.na.2008.02.021

[12] Tarantello, G. (1992) On Nonhomogeneous Elliptic Equations Involving Critical Sobolev Exponent. Annales de l'Institut Henri Poincare. Analyse Non Lineaire, 9, 281-304.

[13] Brown, K.J. and Zhang, Y. (2003) The Nehari Manifold for a Semilinear Elliptic Equation with a Sign Changing Weight Function. Journal of Differential Equations, 193, 481-499. https://doi.org/10.1016/S0022-0396(03)00121-9 
[14] Dr'abek, P., Kufner, A. and Nicolosi, F. (1997) Quasilinear Elliptic Equations with Degenerations and Singularities. Walter de Gruyter Series in Nonlinear Analysis and Applications Vol. 5 (New York). https://doi.org/10.1515/9783110804775 